\title{
Quantification of topographic changes in the surface of back of young patients monitored for idiopathic scoliosis: correlation with radiographic variables
}

Laura Pino-Almero

María Fe Mínguez-Rey

Salvador Sentamans-Segarra

María Rosario Salvador-Palmer

Rosa María Cibrián-Ortiz de Anda

Javier López-de La O 


\title{
Quantification of topographic changes in the surface of back of young patients monitored for idiopathic scoliosis: correlation with radiographic variables
}

\author{
Laura Pino-Almero, ${ }^{a, *}$ María Fe Mínguez-Rey, ${ }^{\text {a,b }}$ Salvador Sentamans-Segarra, ${ }^{a}$ María Rosario Salvador-Palmer, ${ }^{c}$ \\ Rosa María Cibrián-Ortiz de Anda, ${ }^{c}$ and Javier López-de La $O^{c}$ \\ ${ }^{a}$ Clinic University Hospital of Valencia, Department of Orthopedic Surgery and Traumatology, Blasco Ibañez Avenue, Number 17, 46010 Valencia, \\ Spain \\ bUniversity of Valencia, Department of Surgery, Medicine School, Blasco Ibañez Avenue, Number 13, 46010 Valencia, Spain \\ 'University of Valencia, Department of Physiology, Blasco Ibañez Avenue, Number 13, 46010 Valencia, Spain
}

\begin{abstract}
Idiopathic scoliosis requires a close follow-up while the patient is skeletally immature to detect early progression. Patients who are monitored by radiographs are exposed to high doses of ionizing radiation. The purpose of this study is to evaluate if an optic noninvasive method of back surface topography based on structured light would be clinically useful in the follow-up of young patients with idiopathic scoliosis. This could reduce the number of radiographs made on these children. Thirty-one patients with idiopathic scoliosis were submitted twice to radiograph and our topographic method at intervals of 6 months to 1 year. Three topographical variables were applied horizontal plane deformity index (DHOPI), posterior trunk symmetry index (POTSI), and columnar profile (PC). A statistically significant correlation was found between variations of Cobb angle with DHOPI $(r=0.720, p<0.01)$ and POTSI $(r=0.753, p<0.01)$ during the monitoring period. Hence, this topographic method could be useful in clinical practice as an objective adjuvant tool in routine follow-up of scoliosis. () 2016 Society of Photo-Optical Instrumentation Engineers (SPIE) [DOI: 10.1117/1.JBO.21.11.116001]
\end{abstract}

Keywords: scoliosis; adolescent; moiré topography; x-rays; diagnosis.

Paper 160381RR received Jun. 14, 2016; accepted for publication Oct. 12, 2016; published online Nov. 1, 2016.

\section{Introduction}

Scoliosis is a complex three-dimensional (3-D) deformity of the spine that may cause a severe modification of the external appearance of the back and trunk. For this reason, the asymmetry of the back is the main clinical sign of this disease. The affected children and teenagers require a close follow-up until skeletal maturity. The measurement of the lateral flexion angle of the curve (Cobb angle) ${ }^{1}$ in radiographs of spine is currently the gold standard, but this can result in taking $>25$ radiographs during the clinical management of these patients. ${ }^{2}$ Therefore, patients with scoliosis are submitted to high doses of ionizing radiation and exposed to its harmful effects such as leukemia $(0.8 \%)$, breast cancer $(2.1 \%)$, or inherited defects $(3 \%){ }^{3}$

Many nonradiographic and safe methods for the assessment of external morphology of back have been proposed as an alternative to x-rays to avoid its side effects. ${ }^{4}$ Moiré's topography was one of the first techniques. ${ }^{5}$ After that many other methods appeared such as the integrated shape investigation system (ISIS) ${ }^{6}$ ISIS $2{ }^{7}$ photometric $4-\mathrm{D},{ }^{8}$ orthoscan, ${ }^{9}$ or surface dynamic topography. ${ }^{10}$ All these methods apply the projection of multiple rows of light on a subject's back, in such a way that the pattern of the distortion obtained recreates a quantifiable reconstruction of the subject's back and allows the assessment of possible asymmetries by applying a specific software program. The goal of researchers is to substitute or at least minimize the number of radiographs. $\mathrm{x}$-rays would be reserved for cases where the

*Address all correspondence to: Laura Pino-Almero, E-mail: laupialm@yahoo .es topography will show an increment of the back's asymmetry. Other motivations for these methods are the ease of repeating optical examinations and they provide information of changes in back's morphology in the three dimensions of space.

In this study, we used a method based on structured light to obtain the back surface topography of patients in a growth phase with idiopathic scoliosis. The capacity of our method to detect changes of the spine's curve in these patients over time was assessed. The main objective was to quantify the deformity of back and trunk associated with scoliosis in the three planes of space over time and to assess the possible correlation between topographic and radiographic changes in these patients.

\section{Methods}

\subsection{Study Protocol and Data Collection}

It is a prospective study approved by the Research in Humans Ethics Committee of our hospital on May 2009. All parents of patients were informed of the safety of topographic test and they facilitated their approval by signing the corresponding patient consent document.

Thirty-one patients with idiopathic scoliosis, 27 women and 4 men, mean age 13 years (range, 7 to 17 years), referred to our hospital for treatment and monitoring, were evaluated between November 2010 and April 2013. The mean value of Cobb angle was $21.78 \pm 5.46(\mathrm{SD})$ (range, 13.10 to $35.00 \mathrm{deg}$ ). Fourteen patients had a Cobb angle $<20 \mathrm{deg}, 14$ between 20 and $29 \mathrm{deg}$, and 3 subjects $>30 \mathrm{deg}$. Nine patients presented a single

$1083-3668 / 2016 / \$ 25.00$ (c) 2016 SPIE 
curve (1 thoracic; 4 lumbar; 4 thoracic-lumbar) and 22 subjects a double curve. The mean value of body mass index (BMI) was $18.83 \pm 2.25$ (SD) (range, 13.85 to 21.93 ). In 15 cases, there was an increase in the value of the Cobb angle, but only in 6 patients did the increase exceed $5 \mathrm{deg}$.

Inclusion criteria included those patients in a growth phase, diagnosed with juvenile or adolescent idiopathic scoliosis, that presented in physical examination a positive forward bending test and a radiographic Cobb angle equal to or higher than 10 deg. Patients with known etiology of scoliosis such as neuromuscular origin, congenital, tumor, osteochondrodystrophy; subjects that presented a positive Adam test in physical examination, but the Cobb angle was lower than $10 \mathrm{deg}$; or those with missing data were excluded.

\subsection{Patient Evaluation}

Evaluation of the patients consisted of a clinical, radiographic, and topographic examination. These patients were examined every 6 months for early detection of a possible progression of their curve. A chart review was completed to obtain age, sex, how the disease was detected, and personal and family history. For this study, we collected data derived from radiographic and topographic evaluation of these patients on two separate times (6 months to 1-year interval).

Physical examination included the assessment of range of motion of spine; weight and height; asymmetry of shoulders, shoulder blades, and pelvic girdle; leg length discrepancy and the forward bending test (location of the prominence of the back: cervical, thoracic, or lumbar; right or left).

All subjects underwent standard radiography of the entire spine (30 to 90). The lateral flexion angle was determined by means of the Cobb angle method over the anteroposterior projection. We also determined the thoracic kyphosis angle between T4 and T12 vertebrae and the lumbar lordosis angle between $\mathrm{T} 12$ and L5 vertebrae over the profile projection.

Furthermore, all subjects were studied by our back surface topographic method. This allowed obtaining three topographical variables, horizontal plane deformity index (DHOPI), posterior trunk symmetry index (POTSI), and columnar profile (PC), to quantify the back asymmetry in the three planes of space. These variables will be explained in the next section.

\subsection{Back's Surface Topographic Method}

Surface topography consists of the representation of the surface function of an object by means of level curves. An experimentally easy method consisting of the projection of a pattern of preestablished coded colors was used to obtain this surface function. This pattern was formed by 90 vertical parallel lines of three colors, red, green, and blue, forming a sequence of six consecutive lines that did not repeat themselves in the pattern. This allowed locating each line on the back of the patient and on a flat surface of reference.

A mobile white screen with two positions (front and rear); a projector EPSON (3LCD projector model: EMP-835) for the projection of a structured light pattern; a digital camera Canon (1.7 Megapixel, 3LCD Digital Video Camcorder XM2 PAL Video Lens $20 \times$ zoom 4.2 to $84 \mathrm{~mm}$ ) for image capture; and a computer (MacBook Pro) with the program developed in MATLAB 7.9.0, composed the system.

At the beginning of each session, a system calibration must be performed. This is not repeated if the position of the elements is not modified. For that, two images with the color code pattern projected over the white screen, one with the screen in forward position and the other one with the screen in rear position, are captured (Fig. 1). In addition, the distance between two lines separated by a distance corresponding to 20 lines on the screen with the color code pattern projected in the rear position is measured ("rear calibrated distance"). The same distance is measured with the screen in the forward position ("front calibrated distance"). This will get the pattern in both images, identifying each and every one of the points of the pattern on the front network and their equivalents on the rear network.

Once the calibration is made, we proceed to the study of the subject's back. The patient is placed in front of the screen in a rear position and two photographs of the back, one with the color code pattern projected and another one without the pattern projected ("illuminated image"), are taken (Fig. 2). This last

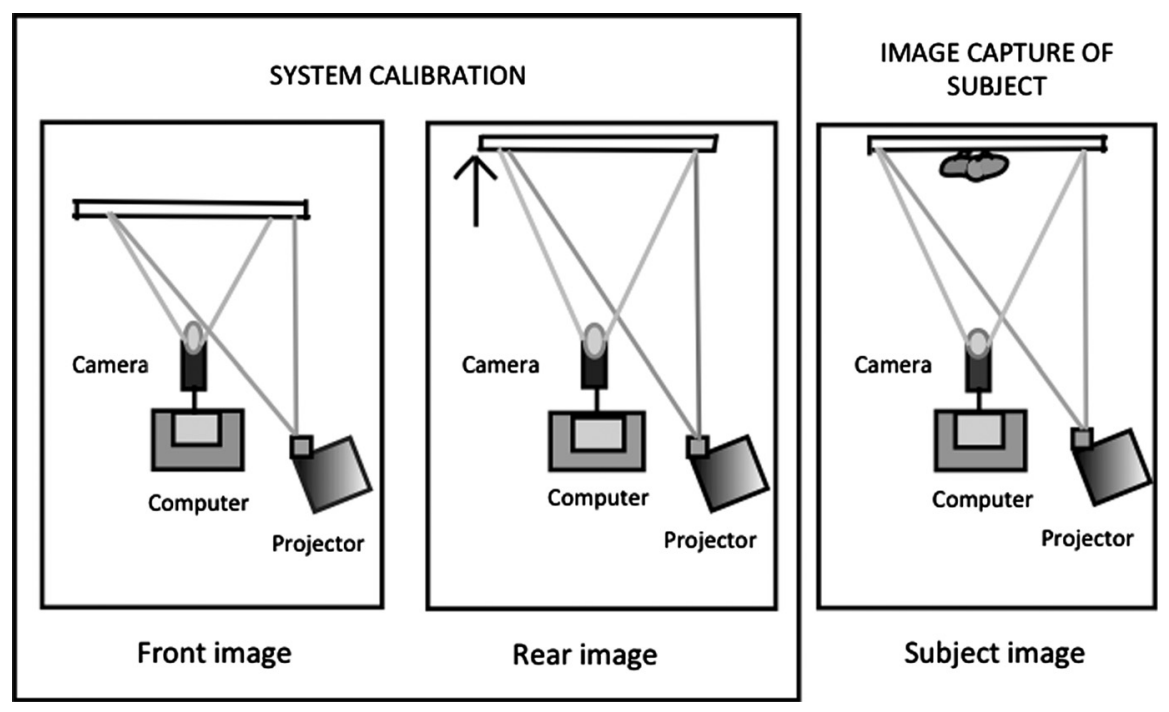

Fig. 1 System calibration and image acquisition. Two images are captured for the system calibration, one with the screen in forward position (front image) and another one with the screen in rear position (rear image). Later, the subject's back image is captured with the screen in rear position. 

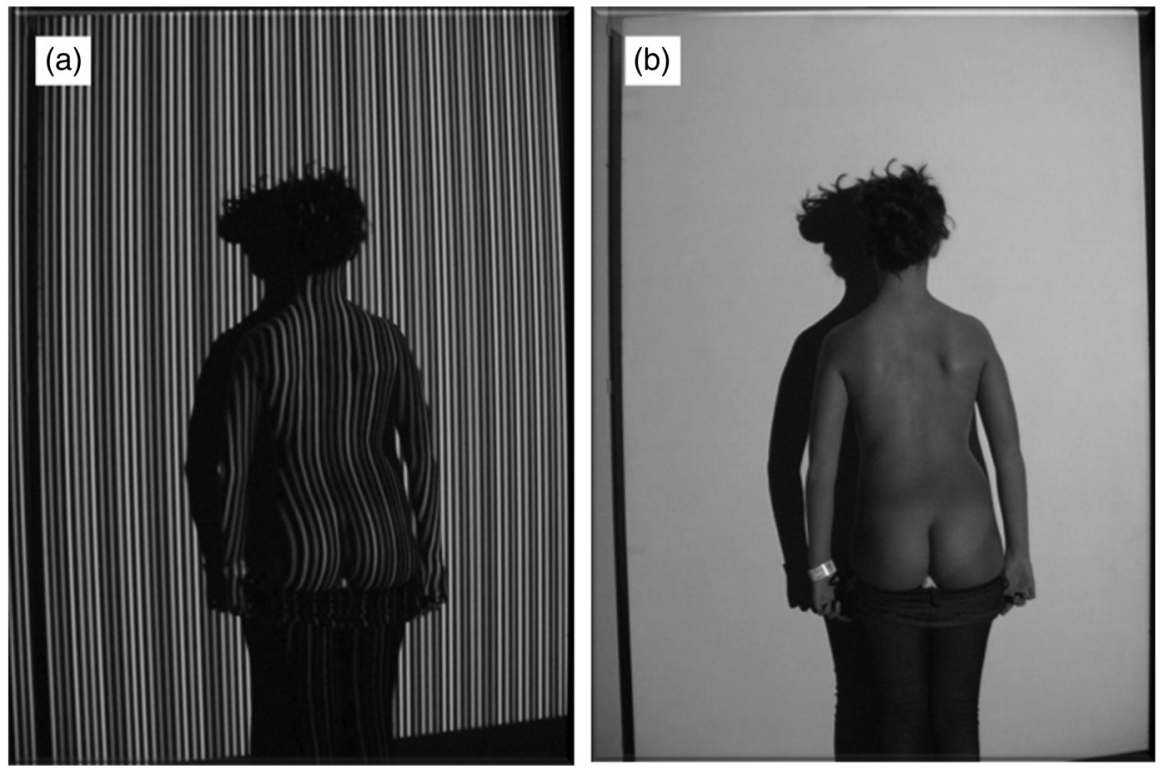

Fig. 2 Correct position of patient and image capture of back's subject: (a) one image with the color code pattern projected. (b) Another one without the color code pattern projected (illuminated image).

image allows better identifying of the boundaries back for further image processing.

A correct position of the patient is essential to avoid errors: the subject must fully expose the back from the neck to the buttocks and should be placed with the back to the camera, placing the feet in a mark attached to the floor, chest, and abdomen slightly contacting the screen; arms should fall relaxed at the sides; and head should be straight with eyes looking straight ahead.

These four captured images are processed through three programs developed in MATLAB 7.0.0 (MATLAB and Simulink Release 2009. Comp.soft-sys.matlab. The MathWorks, Inc., Natick, Massachussetts). The first is called "Calibration." We have to select two points separated by a distance of 20 lines within the image of the "rear position screen" and introduce the distance in millimeters that we have previously measured on the screen at the time of calibration.

Then, the program called "Esc" processes the images and allows obtaining the topographic image of the subject's back.

Finally, the third program, "Point CT," allows obtaining the topographic variables by selecting 16 anatomical landmarks using the mouse as an interface (Fig. 3): 1 to 6 points (corners of the shoulders, right and left; axillary folds, right and left; and pelvic girdle, right and left; respectively) allow calculation of POTSI and are selected on the "illuminated image." 7 to 10 points [7: inter shoulder blade most prominent point of the spine (T5); 8: less prominent lumbar spine point (L3); 9: the start point of the gluteal fold; and 10: point in the neck base level (C7)] allow getting PC. The points 11 to 14 (most prominent points of the shoulder blades, right and left; least prominent points on lumbar pit, right and left, respectively) allow the calculation of DHOPI. 7-14 points are marked on the topographic image because it allows selecting the points depending on their depth. 15 and 16 correspond to two points located at the center of both buttocks placed at the same height. These points allow correcting of poor subject positioning by rotating the image on a vertical axis until both points are at the same depth.

System calibration is performed within $1 \mathrm{~min}$. The capture of the two photo of a patient's back takes another minute. The processing of all images with the software to obtain the topographical image and topographical variables takes $8 \mathrm{~min}$.

\subsection{Topographic Variables}

Three topographic variables were used to quantify the asymmetry of the back surface in all three planes of space:

- POTSI ${ }^{11}$ is the sum of two variables [Fig. 4(a)]: height asymmetry indices (HAI) and frontal asymmetry indices (FAI). HAI is obtained as the sum of height differences of the shoulders (distance F), axillary folds (distance G), and waist creases (distance $\mathrm{H}$ ) and it is normalized with the division of its value by the distance I (the vertical distance from the $\mathrm{C} 7$ vertebra to the baseline of the gluteal cleft). To determine FAI, the differences in horizontal distance with respect to the gluteal cleft, of $\mathrm{C} 7$ vertebra (distance A), axillary folds (B-C), and waist (D-E), are obtained and their sum is calculated, also normalized by dividing them by distance I.

- For calculation of DHOPI, ${ }^{12}$ the software draws two lines [Fig. 4(b)]: (a) The line between the two most prominent points of the scapulae, points $\mathrm{B}-\mathrm{A}$; (b) the line between the two least prominent points of the waist, points D-C. Afterward, it locates the symmetrical point of the most prominent point situated on the two above described lines. Finally, the differences in depth between the symmetrical points, divided by distance I, are added up.

- PC, a variable for quantification asymmetry in the sagittal plane, it is obtained by determining the three angles that are formed when identifying the following points in the topography [Fig. 4(c)]: the first angle (PC1) is delimited by the line between the basis of the neck ( $\mathrm{C} 7$ vertebra) with the intershoulder blade zone (T5 vertebra) and the vertical line. The second angle (PC2) is delimited by the line between the anterior point (T5) with the waist zone (L3) and the vertical line. The third angle (PC3) 
Pino-Almero et al.: Quantification of topographic changes in the surface of back of young patients...

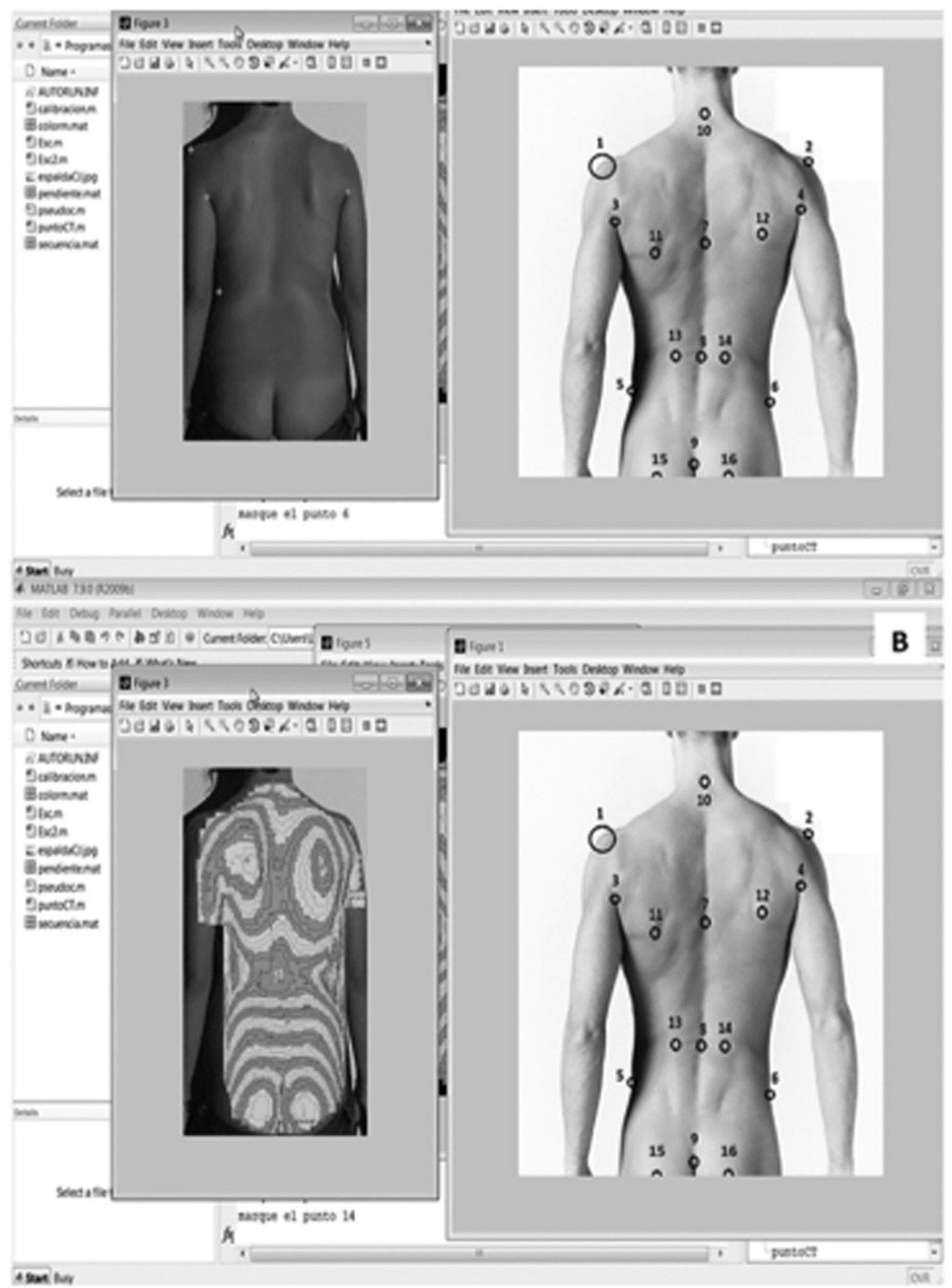

Fig. 3 Sequential selection of 16 anatomical landmarks on the illuminated and topographic image for obtaining topographic variables by means of the program "Point CT" using the mouse as interface.
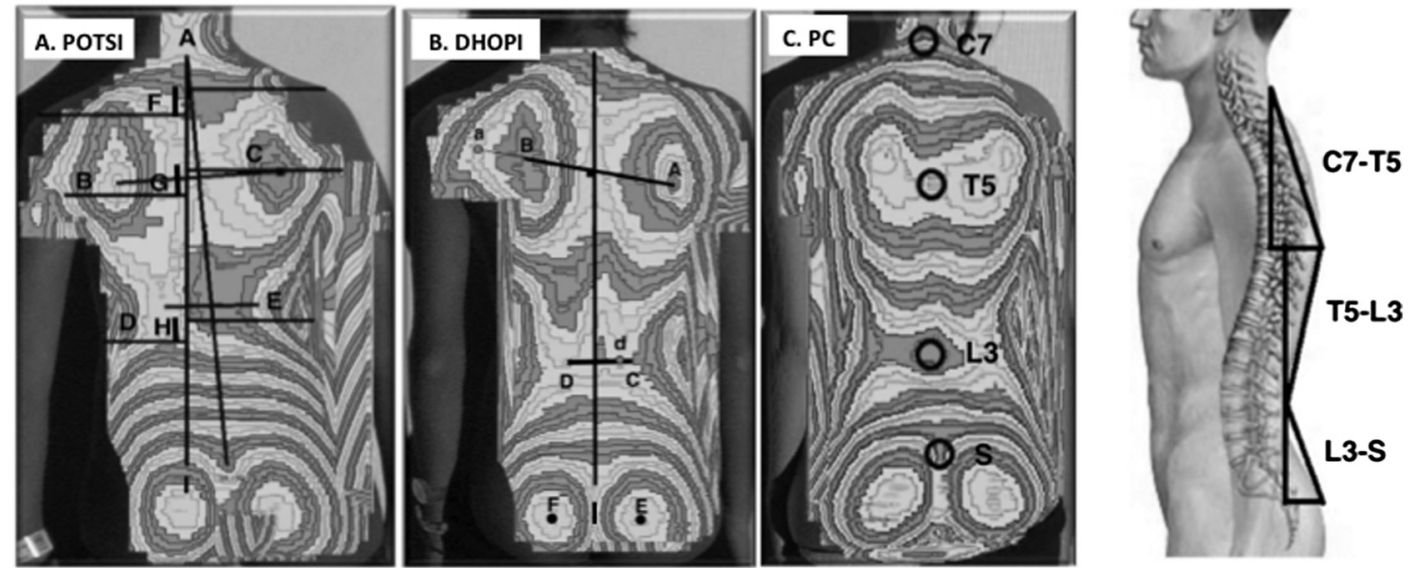

Fig. 4 (a) Theory basis of POTSI. (b) Theory basis of DHOPI. (c) Theory basis of PC and its component angles. 
is delimited by the line between the anterior point corresponding to L3 with the intergluteal cleft (sacrum) and the vertical line.

The software provides the values of the three topographical variables in an Excel spreadsheet, which is saved in a PC folder for each subject with its correspondent topographic images.

A single researcher, a specialist physician in orthopedic surgery trained in this topographic method and its software, performed all measurements, but any clinician with some training with the program can perform the measurement. Furthermore, these measurements were repeated by the same researcher and a second observer to determine the reliability of the method.

\subsection{Statistical Analyses}

Statistical analyses were conducted with IBM SPSS statistics program v.19. First, the interclass correlation index for intraobserver and interobserver measurements was calculated. Descriptive statistics were provided in the form of mean \pm standard deviation (SD). The linear correlation between radiographic and topographical variables was assessed by calculating Pearson's linear correlation coefficient $r$ and the coefficient of determination $\left(R^{2}\right)$. Statistical significance was set at 0.01 .

\section{Results}

The quantification of topography with the software was repeated in the 31 cases by the same observer and by a different observer. The interclass correlation index for intraobserver measurements was 0.983 for DHOPI, 0.959 for POTSI, and 0.984 for PC; for interobserver measurements, it was 0.987 for DHOPI, 0.978 for POTSI, and 0.969 for PC $(P<0.05)$. Therefore, intraobserver and interobserver correlation was excellent.

\subsection{Radiographic Variables}

The mean value of the main curvature's Cobb angle measured at the moment of first evaluation was $21.78 \mathrm{deg} \pm 5.46$ (SD), and $21.97 \mathrm{deg} \pm 6.61$ (SD) at the moment of the second evaluation.
The mean value of thoracic kyphosis angle measured at the beginning of the study was $28.77 \mathrm{deg} \pm 9.75$ (SD), and $28.88 \mathrm{deg} \pm 10.34$ (SD) at the endpoint.

The mean value of the lumbar lordosis angle was $45.56 \mathrm{deg} \pm$ 7.37 (SD) at the baseline and $47.01 \mathrm{deg} \pm 7.81$ (SD) at the endpoint.

Cobb angle was categorized into four groups according to four angular values intervals, as a modification of the Scoliosis Research Society. ${ }^{13} 14$ patients had a Cobb angle $<20$ deg, 14 between 20 and $29 \mathrm{deg}$, and 3 subjects $>30 \mathrm{deg}$. No patient had a Cobb angle $>50 \mathrm{deg}$.

The thoracic kyphosis angle and the lumbar lordosis angle measurements were categorized into three groups according to the range of normal values for thoracic kyphosis from 20 to $45 \mathrm{deg}$ and for lumbar lordosis from 30 to $55 \mathrm{deg}$. 28 patients had normal values for thoracic kyphosis angle; two had hypo kyphosis ( $<20 \mathrm{deg})$; and one showed hyper kyphosis ( $>45 \mathrm{deg})$. 27 patients had normal values for lordosis lumbar angle and 4 had hyper lordosis (>55 deg).

\subsection{Topographic Variables}

The mean value for DHOPI at the moment of the first topography was $6.39 \pm 1.39$ (SD) and at the moment of the second topography was $6.16 \pm 1.85(\mathrm{SD})$.

For POTSI, the mean value at the first topography was $20.37 \pm 8.06$ (SD) and $19.23 \pm 7.46$ (SD) at the second topography.

For PC, the mean value at the first topography was $41.02 \pm$ 9.93 (SD) and $40.89 \pm 10.60$ (SD) at the second topography.

\subsection{Correlations Between Radiographic and Topographic Variables}

We calculated the difference between the initial value (1) and the final value (2) of all variables for each patient.

Table 1 summarizes the values of Pearson's linear correlation coefficient $(r)$ and coefficient of determination $\left(R^{2}\right)$ between topographic and radiographic variables that were statistically

Table 1 Pearson's linear correlation test $(r)$ and coefficient of determination $\left(R^{2}\right)$ between radiographic and topographic variables. Statistical significance 0.01 .

\begin{tabular}{|c|c|c|c|c|c|}
\hline Variable 1 & Variable 2 & $\begin{array}{l}\text { Pearson's linear } \\
\text { correlation }(r)\end{array}$ & $\begin{array}{c}\text { Coefficient of } \\
\text { determination }\left(R^{2}\right)\end{array}$ & $\begin{array}{l}\text { Significance } \\
(p<0.01)\end{array}$ & $\begin{array}{l}95 \% \text { confidence } \\
\text { intervals }\end{array}$ \\
\hline Cobb angle 1 & DHOPI 1 & 0.769 & 0.591 & 0.000 & 0.637 to 0.870 \\
\hline Cobb angle 1 & POTSI 1 & 0.539 & 0.291 & 0.002 & 0.266 to 0.774 \\
\hline DHOPI 1 & POTSI 1 & 0.477 & 0.227 & 0.007 & 0.235 to 0.670 \\
\hline Cobb angle 2 & DHOPI 2 & 0.774 & 0.599 & 0.000 & 0.630 to 0.879 \\
\hline Cobb angle 2 & POTSI 2 & 0.540 & 0.291 & 0.002 & 0.143 to 0.804 \\
\hline Thoracic kyphosis angle 1 & PC 1 & 0.531 & 0.282 & 0.002 & 0.068 to 0.780 \\
\hline Thoracic kyphosis angle 2 & PC 2 & 0.608 & 0.369 & 0.000 & 0.311 to 0.805 \\
\hline Difference Cobb angle (1 and 2) & Difference DHOPI (1 and 2) & 0.720 & 0.519 & 0.000 & 0.531 to 0.862 \\
\hline Difference Cobb angle (1 and 2) & Difference POTSI (1 and 2) & 0.753 & 0.567 & 0.000 & 0.458 to 0.933 \\
\hline Difference DHOPI (1 and 2) & Difference POTSI (1 and 2) & 0.523 & 0.274 & 0.003 & 0.217 to 0.763 \\
\hline
\end{tabular}


Pino-Almero et al.: Quantification of topographic changes in the surface of back of young patients...

(a)
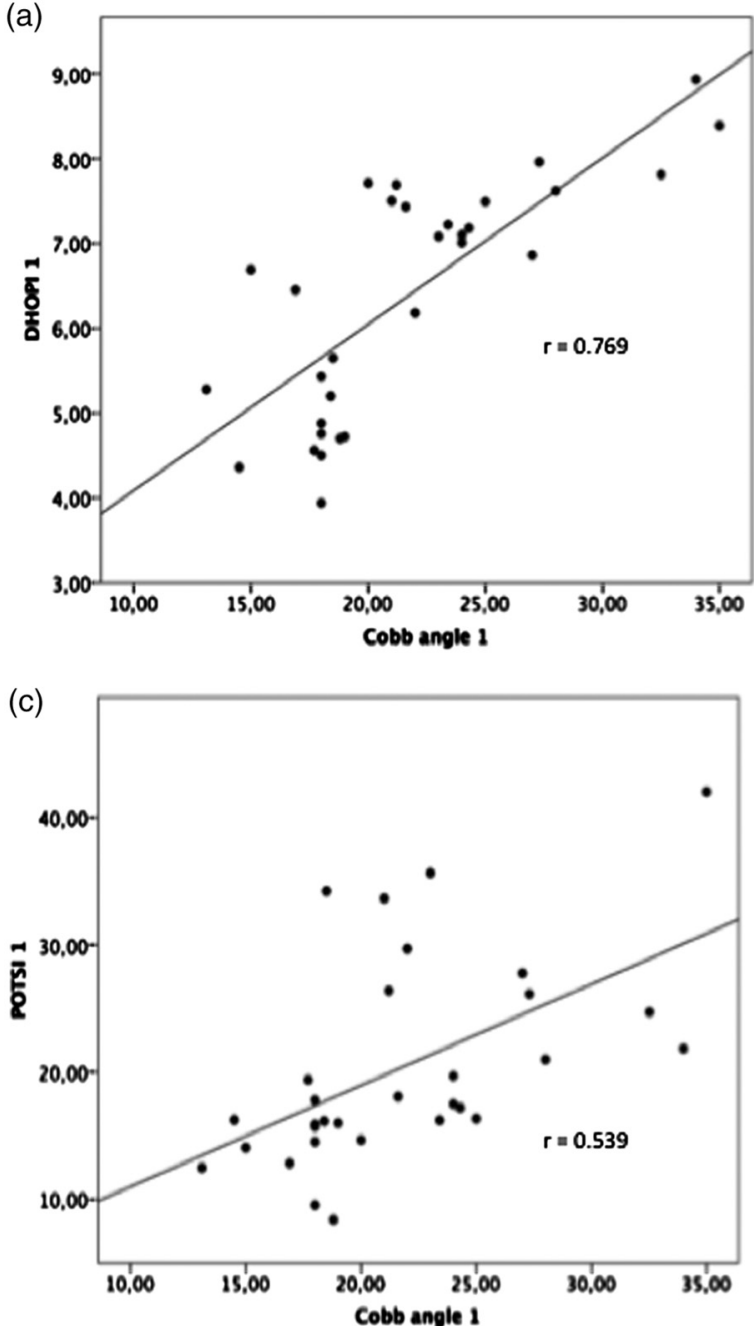

(e)

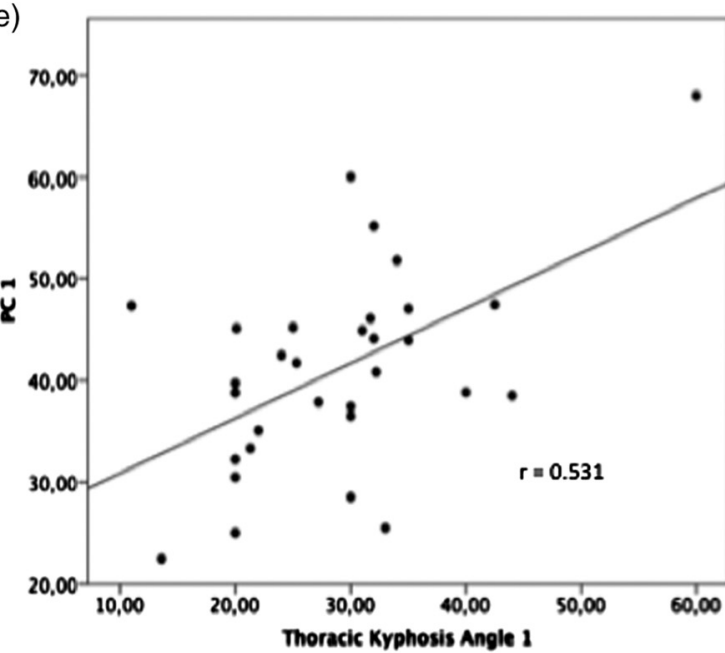

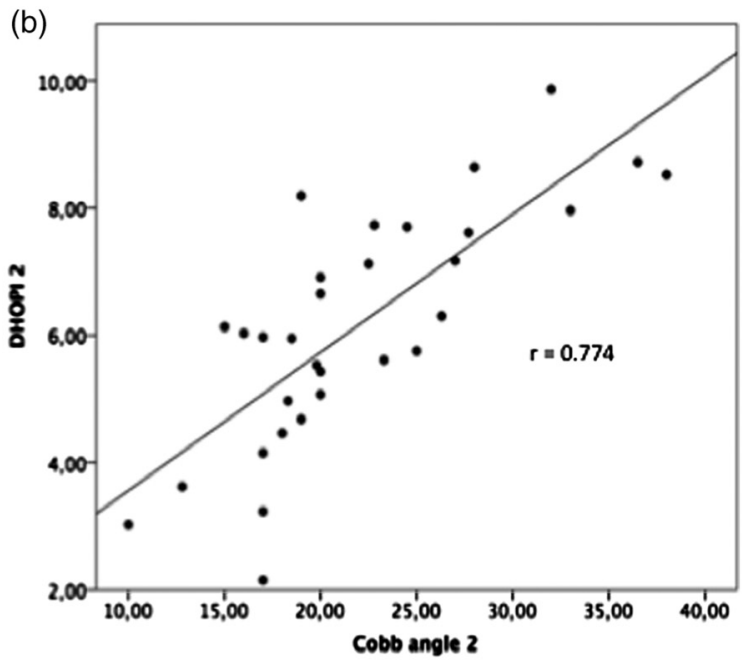

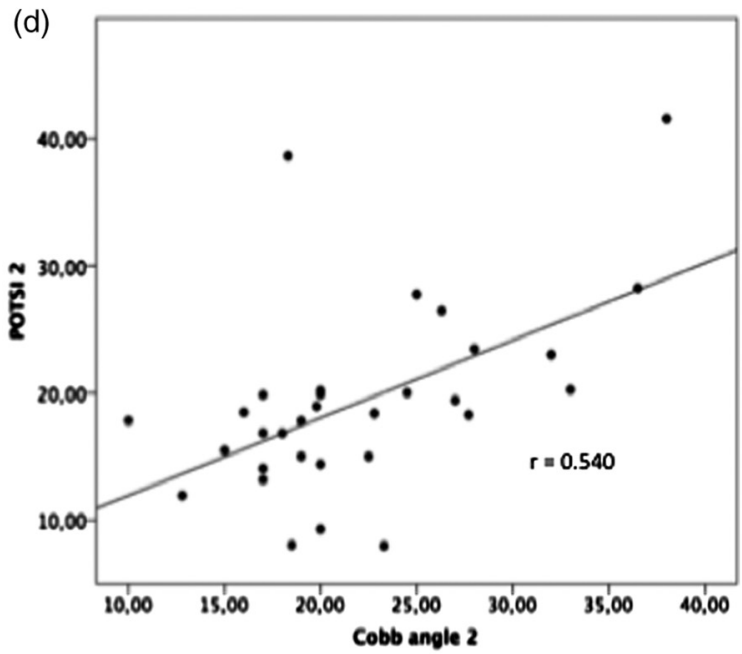

(f)

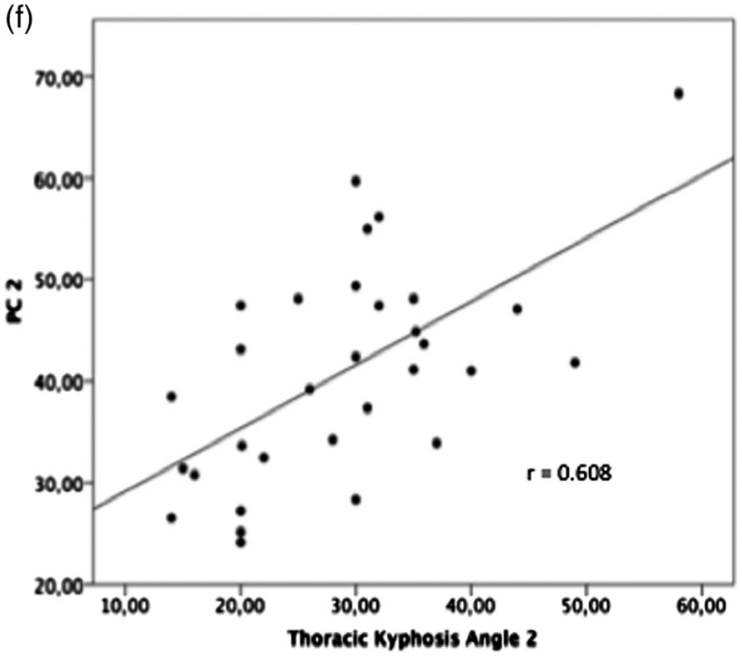

Fig. 5 Scatter plots for the topographic and radiographic variables. The Pearson's linear correlations $(r)$ are also shown $(n=31)$. (a) Correlation between Cobb angle and DHOPI at the moment of first evaluation. (b) Correlation between Cobb angle and DHOPI at the moment of second evaluation. (c) Correlation between Cobb angle and POTSI at the moment of first evaluation. (d) Correlation between Cobb angle and POTSI at the moment of second evaluation. (e) Correlation between thoracic kyphosis angle and PC at the moment of first evaluation. (d) Correlation between thoracic kyphosis angle and PC at the moment of second evaluation. 
significant $(p<0.01)$. It can be seen that there is a statistically significant correlation $(p<0.01)$ between the values of the Cobb angle with DHOPI $\left(r=0.769 ; R^{2}=0.591\right)$ and POTSI $\left(r=0.539 ; R^{2}=0.291\right)$ at the moment of the first evaluation and also at the moment of the second evaluation $(r=0.774$, $R^{2}=0.599$ for DHOPI; and $r=0.540, R^{2}=0.291$ for POTSI). We have also found a statistically significant correlation $(p<0.01)$ between the values of PC and thoracic kyphosis angle in both moments of evaluation $\left(r=0.531, R^{2}=0.282\right.$; and $r=0.608, R^{2}=0.369$, respectively). Finally, a statistically significant correlation $(p<0.01)$ was found between the difference of the Cobb angle (Cobb angle 1 and Cobb angle 2) with the difference of DHOPI (DHOPI 1 and DHOPI 2), $r=0.720$ and $R^{2}=0.519$, and with the difference of POTSI (POTSI 1 and POTSI 2), $r=0.753$ and $R^{2}=0.567$. As well, there is a statistically significant Pearson's correlation between the difference of DHOPI (DHOPI 1 and DHOPI 2) and the difference of POTSI (POTSI 1 and POTSI 2), $r=0.523$ and $R^{2}=0.274$.

Figures 5 and 6 show these correlations. No significant correlation was found between PC and variations of radiographic variables.
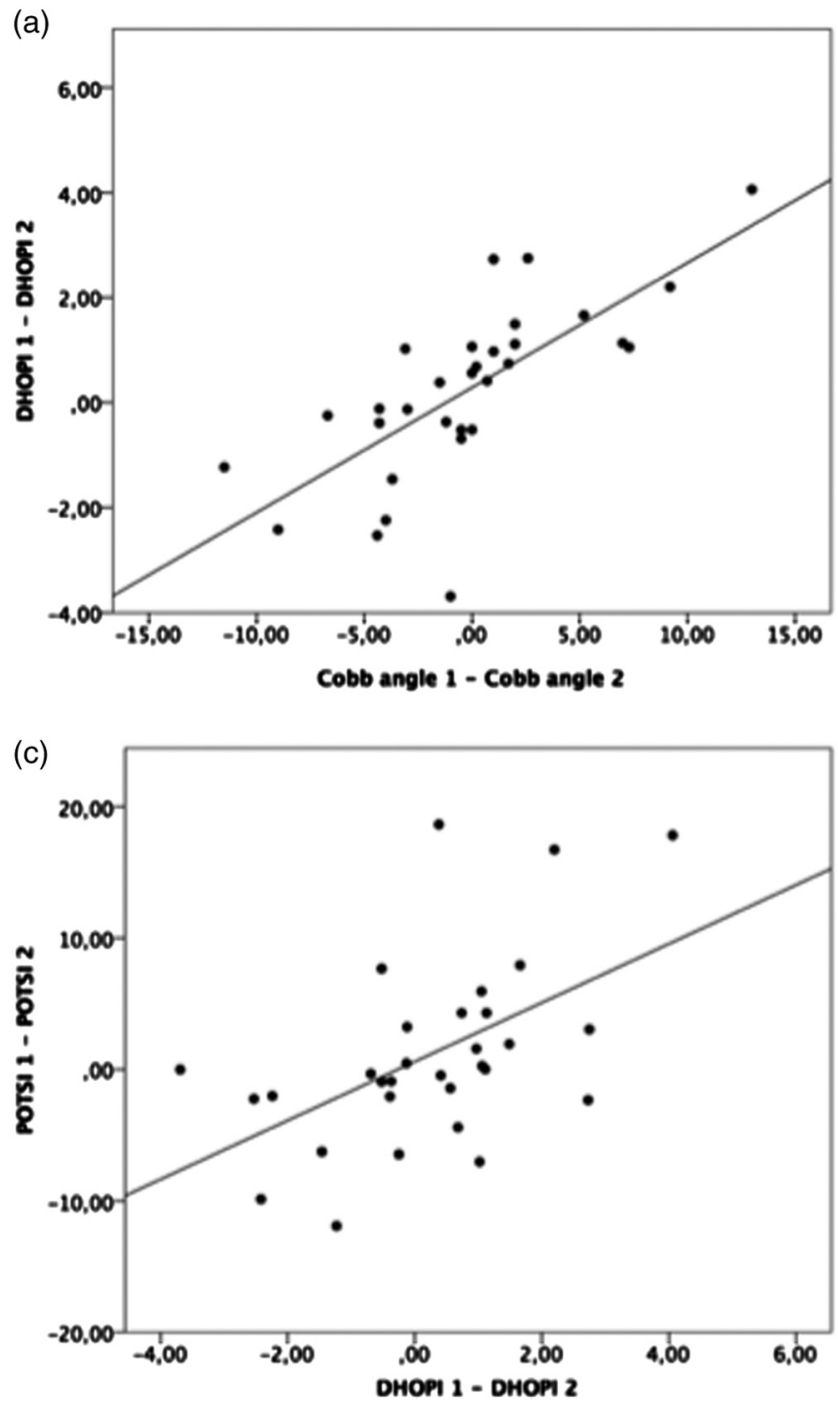

\subsection{Curvature's Progression: Diagnostic Criterion for Topographic Monitoring of Patients with Scoliosis}

Since it is considered that a difference $>5$ deg in Cobb angle obtained in two different radiographs is an evidence of progression in the curvature, we categorized the variation of Cobb angle into three groups:

- Decrease: if the obtained variation showed a $5 \mathrm{deg}$ reduction.

- Increase: if the obtained variation showed a $5 \mathrm{deg}$ increase.

- Invariable: if the obtained variation was $<5 \mathrm{deg}$.

The two topographical variables which were used to assess the possible progression of the curve were DHOPI and POTSI, as these variables were those that showed a higher correlation with the Cobb angle.

As the critical values of these topographical variables for normal control subjects described in the literature are

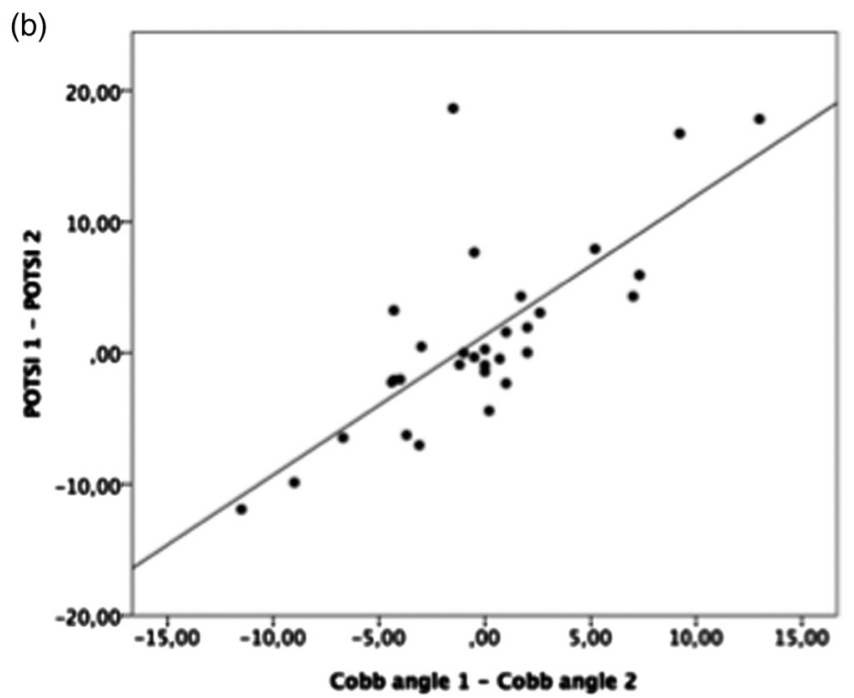

Fig. 6 Scatter plots for the difference of topographic and radiographic variables. The Pearson's linear correlations $(r)$ are also shown $(n=31)$. (a) Correlation between difference of Cobb angle and difference of DHOPI. (b) Correlation between difference of Cobb angle and difference of POTSI. (c) Correlation between difference DHOPI and difference of POTSI. 
$1.44 \pm 0.85$ (mean \pm standard deviation) for DHOPI and $13.47 \pm 5.69$ (mean \pm standard deviation) for POTSI, ${ }^{10}$ we established that changes for DHOPI of 1 point and 5 points for POTSI represented a change in topography, taking into account its standard deviation.

Table 2 shows the distribution of patients according to the variation in Cobb angle and DHOPI variable.

It can be seen that the level of agreement between variations in Cobb angle and variations in DHOPI was 64.50\%. This means that in 20 of 31 patients, when the radiographic variable (Cobb angle) increased, the topographical variable also increased; when the Cobb angle remained stable, DHOPI also remained stable; and when the Cobb angle worsened, the topographical variable also worsened.

Table 3 shows the same but with the POTSI variable. In this case, the level of concordance between variations in Cobb angle and variations in POTSI was $83.87 \%$ (26 of 31 patients).

We evaluated three possibilities of combinations of these two variables, DHOPI and POTSI, in such a way that patients were categorized into three groups:

1. Regression: When both variables decreased during follow-up time, i.e., the value of DHOPI in the second topography decreased 1 point with respect to the initial assessment and at the same time, the value of POTSI decreased 5 points compared to the original measure.

2. Stable situation: When both variables remained stable, or one variable increased and the other one decreased, or only one variable of the two increased or decreased.

3. Progression: When both variables increased during follow-up time. That is, the value of DHOPI increased

Table 2 Distribution of patients according to the difference in Cobb angle and DHOPI variables.

\begin{tabular}{lccccc} 
& & \multicolumn{4}{c}{ DHOPI difference } \\
\cline { 3 - 6 } & & Decrease & Invariable & Increase & Total \\
\hline $\begin{array}{l}\text { Cobb angle } \\
\text { difference }\end{array}$ & Decrease & 2 & 0 & 0 & 2 \\
& Invariable & 4 & 13 & 6 & 23 \\
& Increase & 0 & 1 & 5 & 6 \\
\hline Total & 6 & 14 & 11 & 31 \\
\hline
\end{tabular}

Table 3 Distribution of patients according to the difference in Cobb angle and POTSI variable.

\begin{tabular}{lccccc} 
& \multicolumn{5}{c}{ POTSI difference } \\
\cline { 3 - 6 } & & Decrease & Invariable & Increase & Total \\
\hline $\begin{array}{l}\text { Cobb angle } \\
\text { difference }\end{array}$ & Decrease & 2 & 0 & 0 & 2 \\
& Invariable & 2 & 19 & 2 & 23 \\
& Increase & 0 & 1 & 5 & 6 \\
\hline Total & 4 & 20 & 7 & 31 \\
\hline
\end{tabular}

Table 4 Relationship between topographic and radiographic criterion.

\begin{tabular}{lccccc}
\hline & & \multicolumn{3}{c}{ Topographic variables combination } & \\
\cline { 3 - 5 } & & Worsening & Invariable & Improvement & Total \\
\hline Cobb angle & Increase & 2 & 0 & 0 & 2 \\
difference & & 1 & 22 & 0 & 23 \\
& Invariable & 1 & 2 & 4 & 6 \\
\hline Total & Decrease & 0 & 24 & 4 & 31 \\
\hline
\end{tabular}

in the second topography $>1$ point, and the value of POTSI $>5$ points.

Table 4 shows the distribution of patients according to variation of Cobb angle and the three possibilities of combinations of DHOPI and POTSI.

The level of agreement between both methods-topographic and radiographic-was $90.32 \%$ (28 of 31 patients) with this combined criterion. In this way, the results with the combined topographical criterion are better than with topographical variables individually.

In this way, the role of the physician would be to make a first topography of the patient and to obtain the values of topographic variables. At 6 months from the first test, back surface topography is repeated. The difference in the values obtained for the topographic variables DHOPI and POTSI is calculated. If the obtained result is a stable or in a regression situation, patient observation should continue by topographic controls, and radiographs would not be necessary. But if there were a progression, a radiographic study would be necessary to confirm it and to decide whether to start treatment with a brace according to the Cobb angle. This will avoid making a large number of $\mathrm{x}$ rays because a radiographic study will be realized only when there is a strong suspicion of progression in topography. In our study, we could have avoided making a second radiograph in $70.96 \%$ ( 22 of 31 patients) of cases through the assessment of progress with our topographic method.

\section{Discussion}

The goal of this research is to evaluate if our safe surface topographic method based on structured light could be clinically useful as a complementary tool to physical examination and x-ray to detect possible progression of idiopathic scoliosis, replacing some radiographs during the follow-up to reduce the risks of ionizing radiation in young patients. Few studies in medical literature can be found in which topographic methods have been used in follow-up of patients with scoliosis to evaluate its possible progression.

Parent et al.'s study ${ }^{14}$ reveals that some topographic index (DHOPI, Hump Sum, lordosis angle) may be sensitive to changes during monitoring of scoliosis. Schulte ${ }^{15}$ applied a raster stereographic analysis over the course of follow-up of 16 patients with scoliosis during 8 years and showed an excellent correlation between changes in Cobb angle and changes in lateral deviation or axial rotation. The author recommends the application of this technique every 3 to 6 months, whereas a radiograph is advised only once in a year or every 18 months 
in cases in which is detected a slight progression. Berryman ${ }^{7}$ found a direct linear correlation only for one of the topographic indices (lateral asymmetry index; $r=0.84$ ) after monitoring 30 patients with the ISIS2 system. Theologis ${ }^{16}$ found significant mean differences in only one (transverse asymmetry) surface topographic parameter of ISIS in a sample of 78 patients with 5 deg increases in the Cobb angle after 18 months. Goldberg et al. ${ }^{17}$ used the Quantec Spinal Imaging System in 59 patients to detect a 10-deg Cobb angle scoliosis progression over 1 year and obtained a specificity of $45 \%$ and a sensitivity of $100 \%$. Adankon et al. ${ }^{18}$ investigated the difference between topographic measurements and Cobb angle over time in 30 patients with three clinical visits and curve progression of at least $5 \mathrm{deg}$ was detected with a high sensitivity of $92 \%$ and specificity of $79 \%$. De Korvin et al. ${ }^{19}$ used the BIOMOD ${ }^{\mathrm{TM}}$-L system with 100 patients and the threshold for a significant change was set to $2 \mathrm{deg}$ for gibbosities and $10 \mathrm{deg}$ for sinuosities. The $f$ parameter (the increase in the sum of the gibbosities, or an increase in at least one of the gibbosities) was most sensitive for detecting progression over the tree Coob angle thresholds (with sensitivities of $86 \%, 86 \%$, and $100 \%$ for 3,5 , and $10 \mathrm{deg}$, respectively). Finally, Komeili et al. ${ }^{20}$ studied 100 patients with adolescent idiopathic scoliosis during 1 year. His method to detect thoracic/ thoracic-lumbar curves with progression could correctly classify 58 of 78 of these curves that did not progress and 13 of 19 of those that progressed. His classification tree model to detect lumbar curves with progression correctly identified 7 of 23 of those that did not progress and 6 of 7 of the lumbar curves that progressed. The author considers that $43 \%$ of patients with no evident progression on their curves would not have needed radiographs if they had been submitted to back surface topography evaluation. In our study, 22 patients (70\%) who presented an invariable Cobb angle, topographic variables not changed, although some type of scoliosis curves such as triple curves were not studied, in contrast to Komeili's study that includes all kinds of curves.

Therefore, it can be assumed that possible changes of the radiographic curve are parallel to changes of the back's surface due to their strong interrelation. When our topographic and radiographic measurements of the second examination were compared to first, we found a statistically significant positive correlation, between variation of DHOPI and Cobb angle $(r=0.720, p<0.01)$ and between differences of POTSI and Cobb angle $(r=0.753, p<0.01)$, but no correlation was found between differences of PC and Cobb angle or with its radiographic equivalent (thoracic kyphosis and lumbar lordosis angles). This means that a simple and one-to-one relation between the back's surface and spinal shape does not exist. Therefore, a complex linkage exits between these two entities. Furthermore, a statistically significant correlation was found between Cobb angle with DHOPI $(0.769$ and $0.774, p<0.01)$ and POTSI $(0.539$ and $0.540, p<0.01)$, at the time of the first and the second evaluations. The same happens between the thoracic kyphosis angle and $\mathrm{PC}(0.531$ and $0.608, p<0.01)$.

In our study, a few patients showed dissimilar behaviors concerning variations of the Cobb angle and topographic variables. Only one patient, who showed a stable Cobb angle, had changes (worsening) of DHOPI and POTSI. In two patients, who had a decrease of the Cobb angle, topographic variables did not change. This may be due to the fact that the improvement in Cobb angle was not big enough to induce a change in back asymmetry detectable by topography. Otherwise, Kotwicki et al. ${ }^{21}$ demonstrated that is possible that the brace for the treatment of scoliosis in children may stop the worsening of the back asymmetry but not the progression of the spine curvature. For this clinic/radiological discrepancy, the authors conclude that the evaluation of the results of scoliosis treatment should take into consideration clinical parameters and not only radiological data.

According to this study, the combination of DHOPI and POTSI variables could be useful in monitoring the progression of scoliosis due to its correlation to the Cobb angle, which is the gold standard test in the diagnosis and monitoring of young patients with scoliosis. We cannot declare that back surface topographic method may substitute for radiology completely in the follow-up of these patients. Each method assesses different aspects of the deformity, but by applying the topographic technique, it would be possible to reduce the number of exposures to ionizing radiation. The role of the clinician would be to order an X-ray study in cases where a significant change on surface topography is evident, thus when the DHOPI variable increases by $>1$ unit and the variable POTSI $>5$ with respect to the previous measurement. As Drerup ${ }^{22}$ says, surface topography provides extra and objective information about the back's deformity and the absence of limitations in repeating measurements, because it is a harmless technique, is a great advantage.

Although the PC variable has not been shown to be useful in monitoring of scoliosis in this study, it would be interesting to evaluate its clinical useful by means of studies with patients with alterations in the sagittal plane of the spine such as thoracic hyperkyphosis/hypokyphosis or lumbar hyperlordosis/hypolordosis, because there is a statistically significant correlation between the PC variable and the thoracic kyphosis angle in both the first and second measurements $(r=0.531$ and 0.608 , respectively, $p<0.01$ ).

There are many methods described in the literature for the topographic study of the back with technical differences with our method. One of the first that emerged was the moiré's topography ${ }^{23}$ that is based on the phenomenon of interference produced by the shadows projected by a grid over the back surface. In a normal back, the pattern shadow of moiré is equal in both halves of the back, but in scoliosis, the pattern differs with increasing deformity. As limitations, it presents the difficulty of getting a clear shadow, the inability to distinguish the absolute height of two contour lines if these are not continuous and patient positioning can be a source of error. Our software is able to automatically correct a bad position of the patient.

Another method is the ISIS $^{6}$ that combines video-photogrammetry (VICON system) and the digitization of images of moiré. It is a scanner, consisting of a rotating projector which produces a plane of light which is reflected in a mirror, and a television camera which moves integrally with the projector, so that the system moves through the back of the patient who remains in a standing position. This is a difference with our system in which the components are in a static position except for the white screen that is movable for calibration. Previously, reflective markers must be placed on the spinous processes. This is another difference with our method because we do not use markers on the patient's back. The computer records the line that is being formed to move forward the light beam on the surface of the skin. The surface analysis is made from the transverse curves of the back surface passing through these markers. The parameter used for this system is the lateral asymmetry. A 
difficulty of this method is that the placement of the markers is not easy and requires a specialist to avoid errors.

ISIS 2 (Ref. 7) is an improved system based on the original ISIS. One advantage is that no interpolation between the locations of the fringes is needed. The projector is mounted directly above the camera and projects a horizontal fringe pattern onto the patient's back. In our system, the camera and projector are spaced at an angle of $45 \mathrm{deg}$ to each other and we used a vertical stripes pattern. A patient stand (a black tubular framework, arm rests, and footplate) is used to help minimize postural variations and provide the reference plane. Also, stickers are needed to mark bony landmarks (vertebra prominens, lumbar dimples, and spinous processes) on the patient's back. According to Berryman, its use is limited in the case of extremely obese or very muscular patients, because the identification of bony landmarks is more difficult, and in patients with congenital curves with little rotation. Another disadvantage is that it requires an experienced operator to properly locate bony landmarks.

The quantec spinal image system ${ }^{24}$ uses a stripe pattern projected on the subject's back, but this system also utilizes stickers, which are placed on each spinous process from T1 to L5, including the two postero-superior iliac spines. Computer software reconstructs the surface representation using 250.000 points and indicates a line along the spinal posterior elements. The entire system, if required, can be easily modulated and disassembled for transport. Our system can also be transported.

The Formetric 4-D system ${ }^{8,25}$ projects lines of white light ("raster lines") on the back of the patient and captures a digital photo image. This is a difference with our system because our projected pattern is formed by color lines, which improves image quality. The machine then compares the surface topography obtained with a database of thousands of radiographic and topographical characteristics of patients with scoliosis measures, using a complex algorithm to quickly recreate a 3-D representation of the patient's spine. The generated model can be used to calculate the Cobb angle.

Orthoscan and Ortelius 800 (Ref. 9) use a low-intensity electromagnetic field. With a sensor on the finger, the examiner, palpating the spine, records the location of the spinous processes. The system instantly creates a graphic reconstruction of the spine showing the calculated angle of deformity (Cobb angle), the difference in length of the lower limbs and other additional data from the patient's body balance.

The dynamic surface topography developed by Shannon ${ }^{10}$ is a technology based on an optical motion capture device, which generates a 3-D images sequence and provides measures resulting from changes in the position previously marked anatomical landmarks and of the back surface topography.

The BIOMOD ${ }^{\mathrm{TM}} \mathrm{L}_{\text {system }}{ }^{26-28}$ consists of a mobile optical data acquisition system (high-definition camera, inclinometers, and a structured light projector) that also needs anatomical landmarks in spinous processes as well as the sacral dimples. This method, as ours, evaluates the three planes: frontal, sagittal, and transverse. The examiner must validate the automatic location of the anatomical landmarks and the spinous processes and them the different parameters are calculated automatically with the exception of the gibbosity measurements, which necessitates complementary manual intervention. This system provides the following measurements: Cobb's angles, T1 to T12 kyphosis and $\mathrm{L} 1$ to $\mathrm{S} 1$ lordosis angles, axial vertebral rotation, and pelvic parameters.
The CQ electronic system is a portable raster stereography device, ${ }^{29}$ in which the clinician also must mark anatomical landmarks (spinous processes $\mathrm{C} 7$ to $\mathrm{S} 1$ and posterior superior iliac spines). Another difference with our method is that the child is sitting with a forward flexion of the trunk. The maximal value of the trunk rotation is named the surface trunk rotation (STR) and is automatically picked up the dedicated software as the highest rotation value of 19 spine levels.

The method used by Michonski et al. ${ }^{30}$ consists of projecting a set of sinusoidal and gray code images on the surface of the object, capturing the deformed patterns using a digital camera and transforming the obtained values to real coordinates with a calibration prior to the measurement. The data obtained is in the form of raw point clouds. Each point is characterized by its Cartesian coordinates, color, normal vector of the surface, and quality factor. These data form a set of information that fully describes the geometrical properties and color of the measured surface. Similar anatomical structures that are marked in our method must be selected with this system: vertebral prominence of $\mathrm{C} 7$, top of the intergluteal furrow, shoulders, axilla, waist, posterior superior iliac spines, inferior angles of scapula.

Finally, some researchers have recently demonstrated the feasibility of using 3-D ultrasound for the evaluation of scoliosis. ${ }^{31}$ Scolioscan is a newly developed system targeted for scoliosis assessment in clinics by using coronal images of spine generated by a 3-D ultrasound volume projection imaging method. According to these authors, this method appears promising in screening large numbers of patients for progress monitoring and evaluation of treatment outcomes.

Hence, our proposed method does not rely on manually placed markers and thereby eliminates the measurement variation associated with error in marker placements; not relying on marker placements also facilitates the implementation of the method in the clinic. We use a color code pattern with 90 vertical lines forming sequences of 6 consecutive lines that do not repeat themselves in the pattern. This has improved the quality of the topographic image and the accuracy in locating the coordinates of each point of the subject surface. Our image recognition software has a fast algorithm and is simple to use by localizing and systematic signalling of specific points on images of the subject's back to automatically obtain the topographical variables. The main advantages of our topographic method are the accuracy of the 3-D assessment; the harmlessness, therefore, the test can be repeated as often as necessary, and the possibility of data storage. Moreover, this is an inexpensive system because once the physician has the elements, more cost is not necessary.

Regarding the possible limitations of the topographic method, we found obesity to be a limitation as it can mask the asymmetry of the external morphology of the back. In our study, all patients had a BMI lower than 25; therefore, the accuracy of the method for overweight subjects has not been studied. Another limitation described is the variation of positioning of the subject at the moment of image capture because even small variations may produce striking effects on the contours' line pattern and thus on the perception of the back shape. To avoid this error, a strict protocol for patient positioning was established. Furthermore, the two points located in the most prominent area of the buttocks were used to correct, if necessary, the incorrect placement of the subject. However, the repeatability of the method has not been studied to determine the sensitivity of the system as a function of changes in the patient posture or position. Another limitation that could have affected the outcome of 
the topographic evaluation is the accuracy in the identification of the anatomic landmarks. Clinicians need training to use this method, but the marking of anatomical points is made easier with an "illuminated image" of the patient's back. This enables clear identification of the edges of the back's silhouette and anatomical points. The topographic image enables the selection of the points depending on the depth indicated by the level curves. Another limitation is that our method is not able to identify the number of scoliotic curves present in the same patient and is only limited to a special posture at the time of surface topography acquisition, so it cannot be used for the current surface topography images in the database with different patient postures.

\section{Conclusions}

We can conclude that an important degree of correlation has been found between variations of DHOPI, POTSI, and Cobb angle. A combined criterion has been established with two topographic variables, DHOPI, and POTSI. This combined topographic criterion showed a $90.32 \%$ agreement with the radiographic method in the follow-up of our patients. We consider that our back surface topographic method may be clinically useful to assess changes in the external morphology of the back of young patients with scoliosis, providing objective additional information to radiographic studies. These preliminary results suggest that it could be necessary to increase the number of patients in future researches. An increment in the number of topographies applied to each patient for a longer period of time may be other modifications to consider in the design of future studies to obtain more conclusive results.

\section{References}

1. J. R. Cobb, "Outline for the study of scoliosis," in Instructional Course Lectures, Vol. 5, The American Academy of Orthopedic Surgeons, Ed., pp. 261-275, J. W. Edwards, Ann Arbor (1948).

2. M. Doody et al., "Breast cancer mortality after diagnostic radiography: findings from the U.S. Scoliosis Cohort Study," Spine 25, 2052-2063 (2002).

3. C. M. Bone and G. H. Hsieh, "The risk of carcinogenesis from radiographs to pediatric orthopedic patients," J. Pediatr. Orthop. 20, 251254 (2000).

4. N. J. Oxborrow, "Assessing the child with scoliosis: the role of surface topography," Arch. Dis. Child 83, 453-455 (2000).

5. H. Takasaki, "Moiré topography," Appl. Opt. 12, 845-850 (1973).

6. A. R. Turner-Smith, "A television computer 3D surface shape measurement system," J. Biomech. 21, 515-529 (1988).

7. F. Berryman et al., "A new system for measuring three-dimensional back shape in scoliosis," Eur. Spine J. 17, 663-672 (2008).

8. L. Guidetti et al., "Intra- and interday reliability of spine rasterstereography," Biomed. Res. Int. 2013, 745480 (2013).

9. P. Knott et al., "Electromagnetic topographical technique of curve evaluation for adolescent idiopathic scoliosis," Spine 31, E911-E915 (2006).

10. T. M. Shannon, "Development of an apparatus to evaluate Adolescent Idiopathic Scoliosis by dynamic surface topography," Stud. Health Technol. Inform. 140, 121-127 (2008).

11. K. Inami et al., "Analysis of posterior trunk symmetry index (POTSI) in scoliosis. Part 2," Research into Spinal Deformities, Studies in Health Technology and Informatics 59, 85-88 (1999).

12. M. F. Mínguez et al., "Quantifier variables of the back surface deformity obtained with a noninvasive structured light method: evaluation of their usefulness in idiopathic scoliosis diagnosis," Eur. Spine J. 16, 73-82 (2007).

13. E. C. Parent et al., "The association between scoliosis research society22 scores and scoliosis severity changes at a clinically relevant threshold," Spine 35, 315-322 (2010).
14. E. C. Parent et al., "Identifying the best surface topography parameters for detecting idiopathic scoliosis curve progression," Stud. Health Technol. Inform. 158, 78-82 (2010).

15. T. L. Schulte et al., "Raster stereography versus radiography in the longterm follow-up of idiopathic scoliosis," J. Spinal Disord. Tech. 21, 23-28 (2008).

16. T. N. Theologis et al., "Early detection of progression in adolescent idiopathic scoliosis by measurement of changes in back shape with the integrated shape imaging system scanner," Spine 22, 1223-1227 (1997).

17. C. J. Goldberg et al., "Surface topography, Cobb angles, and cosmetic changes in scoliosis," Spine 26, E55-E63 (2001).

18. M. M. Adankon et al., "Scoliosis follow-up using noninvasive trunk surface acquisition," IEEE Trans. Biomed. Eng. 60, 2262-2270 (2013).

19. G. De Korvin et al., "Detection of progressive idiopathic scoliosis during growth using back surface topography: a prospective study of 100 patients," Ann. Phys. Rehabil. Med. 57, 629-639 (2014).

20. A. Komeili et al., "Monitoring for idiopathic scoliosis curve progression using surface topography asymmetry analysis of the torso in adolescents," Spine J. 15(4), 743-751 (2015).

21. T. Kotwicki et al., "Discrepancy in clinical versus radiological parameters describing deformity due to brace treatment for moderate idiopathic scoliosis," Scoliosis 2, 18 (2007).

22. B. Drerup, "Rasterstereographic measurement of scoliotic deformity," Scoliosis 9, 22 (2014).

23. F. Porto et al., "Moiré topography characteristics and clinical application," Gait 32, 422-424 (2010).

24. X. C. Liu et al., "Functional classification of patients with idiopatic scoliosis assessed by the Quantec system: a discriminant functional analysis to determine patient curve magnitude," Spine 26, 1274-1279 (2001).

25. M. Betsch et al., "Radiation-free diagnosis of scoliosis: an overview of the surface and spine topography," Orthopade 44, 845-851 (2015).

26. M. De Sèze et al., "Inter-observer reproducibility of back surface topography parameters allowing assessment of scoliotic thoracic gibbosity and comparison with two standard postures," Ann. Phys. Rehabil. Med. 56, 599-612 (2013).

27. A. Hocquelet et al., "Patient-specific 3D models created by 3D imaging system or bi-planar imaging coupled with Moiré-Fringe projections: a comparative study of accuracy and reliability on spinal curvatures and vertebral rotation data," Eur. Spine J. 25, 3154-3161 (2016).

28. V. Bobin et al., "Monitoring of progressive idiopathic scoliosis by BIOMOD TM-L, back surface topography device, during plaster treatment and progressive felting," Ann. Phys. Rehabil. Med. 59S, e91-e92 (2016).

29. J. Chowanska et al., "School screening for scoliosis: can surface topography replace examination with scoliometer?," Scoliosis 7, 9-15 (2012).

30. J. Michonski et al., "Automatic recognition of surface landmarks of anatomical structures of back and posture," J. Biomed. Opt. 17, 056015 (2012).

31. Y. P. Zheng et al., "A reliability and validity study for Scolioscan: a radiation-free scoliosis assessment system using 3D ultrasound imaging," Scoliosis Spinal Disord 11, 13-28 (2016).

Laura Pino Almero finished her studies in medicine at the University of Valencia in 2002 and was trained as a specialist in orthopedic surgery and traumatology at the Clinical Hospital of Valencia between 2003 and 2008. Currently, she is a part of the pediatric orthopedics unit of this hospital. In 2014, she defended her thesis titled "Clinical application of back surface topography based on structured light through the screening, diagnosis, and monitoring of deformities of the spine."

María Fe Mínguez-Rey was a specialist in orthopaedic surgery at the University Clinical Hospital of Valencia, Spain, and is currently head of the pediatric orthopedics unit in that hospital. She completed her Doctoral Thesis in 2002 under the title "Valuation of structured light techniques in determining spine deformities" at the University of Valencia.

Salvador Sentamans-Segarra was formed as nurse in the Catholic University of Valencia. He has worked throughout the project of study of back surface topography. He currently works at the University Clinical Hospital of Valencia, Spain, and is doing a Master of Emergency Nursing. 
Pino-Almero et al.: Quantification of topographic changes in the surface of back of young patients...

María Rosario Salvador-Palmer is currently professor in the Department of Physiology of the Faculty of Medicine of the University of Valencia, Spain. She holds a PhD in physics. She has participated in many projects related to biophysics and biomedicine. She has made lots of publications and has directed numerous doctoral theses.

Rosa María Cibrián-Ortiz de Anda is professor in the Department of Physiology of the Faculty of Medicine of the University of Valencia, Spain. She holds a PhD in physics. She has participated in many projects related to biophysics and biomedicine. She has directed, together MR Salvador-Palmer, multiple lines of research in the field of biomedicine and has directed numerous doctoral theses.

Javier López-de la $\mathbf{O}$ holds a degree in physics. He completed his doctoral thesis in 2012 under the title "Development of a non-harmful method based on structured light projection, for assessment and help diagnose of spinal deformities" at the University of Valencia. He has worked as an intern in the Department of Physiology of the Faculty of Medicine of the University of Valencia, collaborating in conducting multiple projects. 